\title{
HORIZONTAL AND VERTICAL ZONE BASED LOCATION TECHNIQUES FOR WIRELESS SENSOR NETWORKS
}

\author{
M. Bala Krishna ${ }^{1 *}$ and M. N. Doja ${ }^{2}$

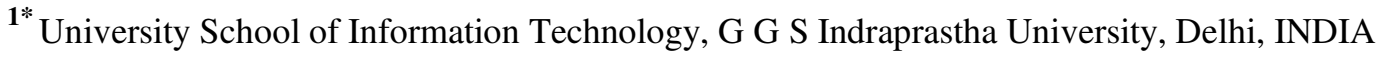 \\ Corresponding Author Email: mbalakrishna@yahoo.com \\ ${ }^{2}$ Department of Computer Engineering, Jamia Millia Islamia, Delhi, INDIA \\ Email: mndoja@gmail.com
}

\begin{abstract}
Localization is an important feature in Wireless sensor networks (WSNs). Accuracy in node localization with proper synchronization and required localization of sensor nodes, save node energy and enhance the performance of communication network protocols. In this paper we propose distributed localization algorithms and assume position known Cluster Head $(\mathrm{CH})$ and position unknown three beacon nodes for each cluster. Using trilateration technique beacon nodes are located. Additional beacon node is added to confirm the location of beacon nodes and maintain location accuracy. These position localized beacon nodes help to locate other sensor nodes. The proposed two distributed zone based localization algorithms are (i) Horizontal Location Position System (H-LPS), where cluster is divided into Horizontal Zones $(\mathrm{HZs})$ and beacon nodes locate in horizontal direction and (ii) Vertical Location Position System $(V-L P S)$, where cluster is divided into Vertical Zones (VZs) and beacon nodes locate in vertical direction. The main advantage of zone based localization is nodes belonging to a bounded zone (horizontal or vertical) are localized and participate in WSN computing. If a bounded zone is eliminated during localization, then nodes do not participate in localization and thus save WSN computing. We provide zone based simulations for H-LPS and V-LPS in comparison with existing localization algorithms like Ad hoc Positioning System (APS), Recursive Positioning Estimation (RPE) and Directed Positioning Estimation (DPE). Performance evaluation of H-LPS and V-LPS illustrate that for zone based localization, H-LPS and $V$-LPS perform better that existing localization techniques. Bounded zone performance is optimal for H-LPS and V-LPS compared to existing localization techniques in WSN.
\end{abstract}

\section{KEYWORDS}

RSSI, Trilateration, Multilateration, Bounded-Box, Time of Arrival (TOA), DV-Hop (Distance Vector), DV-Distance, Cluster Head (CH), Beacon Node (BN), Horizontal Zone (HZ) and Vertical Zone (VZ).

\section{INTRODUCTION}

WSN localization techniques [1] are used to locate sensor nodes with the support of reference nodes and anchor nodes, whose location positions are known. Firstly, nodes are localized using measurements like distance, time of arrival, time difference of arrival, angle of arrival, direction of arrival and communication range [2]. Secondly, position computation techniques [3] like trilateration, multilateration, triangulation, probabilistic approach and bounded box are used to compute position of a node. Finally, centralized or distributed localization algorithms are used to locate WSN nodes. GPS is used for node localization using global coordinate system. WSN localization algorithms are classified [4] as (i) Centralized algorithms and (ii) Distributed algorithms. In Centralized Localization Algorithms, nodes are localized with central located base station with in a given radius. Distance estimation values between nodes are sent to the central base station, where locations of each node are computed. $\mathrm{CH}$ maintains location parameters of all nodes and corresponding network topology. Therefore, centralized localization algorithms optimally compute node positions due to less dense structure and short range distance from $\mathrm{CH}$. The main drawback is traffic congestion caused by node density, and location parameters exchange between nodes and $\mathrm{CH}$ that affect the performance for large scale 
WSN. Hence, distributed localization techniques are used for large scale WSN. Primary centralized localization algorithms in WSN are (i) Convex Position Estimation [5] where nodes are localized with communication constraints between nodes. RSSI (Received Signal Strength Indication) is computed with in a convex region and (ii) Multi-Dimensional Scaling (MDS) [6] where quantitative data and node parameters with Euclidean geometrical parameters are used to compute node distance [7]. Hierarchical MDS (HMDS) based localization algorithm for WSN is given in [8]. In Distributed Localization algorithms, location of $\mathrm{CH}$ and reference nodes helps to locate other nodes in an iterative manner. These algorithms are self-organizing and depend on parameters like node energy, direction of localization and minimum distance from beacon nodes. Network parameters that affect the performance of distributed localization are node density and network topology. Node density is defined as number of nodes per square meter which is directly proportional to localization process. Conversely, localization error in beacon nodes gets propagated if there are more nodes per square meter. Therefore, solution would be to re-compute location parameters of beacon nodes if average percentage of data loss is below a threshold value. Communication parameters that affect the performance of localization techniques are Radio Frequency (RF) channel. Parameters used in RF estimation are Time of Arrival (TOA), RSSI [9], and Angle of Arrival (AOA). Distributed range-based localization techniques in WSN are classified as direct range [10] and extended range [11] [12]. Distributed position-based localization techniques in WSN is given as multi-hop localization, ad hoc position localization, recursive position estimation, direction position estimation and mobile beacon assisted localization. In this paper, we propose algorithms for distribute position-based localization technique. This paper is organized as follows. Section 2 gives overview of related work in distributed localization algorithms for WSN. Section 3 explains localization parameters in our implementation. Section 4 explains in detail our proposed H-LPS algorithm and gives its analysis. Section 5 explains our proposed V-LPS algorithm and gives its analysis. Section 6 gives simulation results, comparative analysis with existing localization techniques and performance of H-LPS and V-LPS techniques. Section 7 concludes our discussion.

\section{RELATED WORK}

Distributed localization algorithms are mainly beacon-based, and calculate node positions from known beacon positions. Beacon nodes are located using GPS system or a priori computed. Various types of beacon-based localization algorithms used in WSN are bounded box, diffusion and APIT. Bounding box [13] uses several beacon nodes, and nodes belonging to intersection of bounded box are used to locate nodes. Diffusion uses centroid of beacon nodes with RSSI to compute location of unknown nodes. Approximate Point In Triangle (APIT) [14] uses area based techniques with more number of beacon nodes to locate a node. Nodes [9] are localized with respect to signal strength and coverage area of beacon nodes. Beacon nodes exchange information across other beacon nodes using Distance Vector (DV) and Distance Hop (DH) techniques. APS [11] uses three beacon nodes and trilateration technique to compute node positions. DV-Hop, DV-Distance and Euclidean geometrics are used to locate nodes. In DV-Hop, beacon nodes exchange information with other beacon nodes using hop count and time stamps. Node exchange information bi-directionally, computes average hop count and calculates respective locations. In DV-Distance, beacon nodes exchange information with other beacon nodes using distance and time stamps. DV-Distance uses RSSI to find average distance. Beacon nodes exchange information bi-directionally, compute average distance and calculate respective locations. In Euclidean technique, APS uses at least two nodes whose locations are known. These two nodes send their location position and distance to unlocalized beacon node. Using geometric parameters like angle and distance between beacon nodes, location position is calculated. This localized beacon node and other reference node help to locate sensor nodes. The main advantage of APS is less number of beacon nodes, which can lead to error position computation. RPE [10] uses localized beacon nodes to identify unknown nodes. Atleast three 
beacon nodes are used to identify unknown node. First, localized beacon nodes are identified. Using RSSI, unknown nodes exchange information with known beacon nodes. Finally, using trilateration unknown node positions are calculated and position parameters are broadcast to neighboring nodes. These localized nodes function as beacon node to locate other sensor nodes. This process is repeated recursively until all the nodes are located. The main advantage of RPE is increase in node localization due to position known beacon nodes. Any error in localized beacon nodes gets propagated and performance of RPE degrades. DPE [15] is variation of REP with restrictions on localization path. DEP localization follows from single point recursion to determined directed path with only two reference nodes. [16] Describes various types of localization techniques. Challenges and current approaches in WSN localization are given [17].

\section{Localization Parameters USED}

\subsection{Distance Computation}

The distance estimation using Time of Arrival (ToA) is classified as:

- One-Way control exchange using DV and time stamps

- Two-Way control exchange using DV and time stamps

- Multipath-Way control exchange using DV and time stamps

In our proposed algorithm we use two-way control exchange to locate a node. For node distance confirmation, we add a beacon node and use multipath-way control exchange to confirm node position. We use TOA since minimum numbers of signals are used between nodes to compute node distance. Distance between two nodes is proportional to signal propagation. If a signal $\mathrm{S}$ is sent at time $t 1$ and received at time $t 2$, then distance between two nodes is calculated as:

$$
d=\text { Signal_Propagation_Speed } *(t 2-t 1)
$$

where signal propagation speed is given in Kbits/sec over single hop or multi hop. TDoA uses combination of signals, $S 1$ and $S 2$ to estimate node distance.

\subsection{Position Computation}

Node position is computed using absolute, relative or global coordinates. Various methods used in node position computation are trilateration, multilateration, triangulation, bounded box and probabilistic approaches. In our proposed algorithm, we use trilateration and multilateration techniques. In Trilateration, $\mathrm{CH}$ functions as reference node with known position (x, y). Trilateration uses intersection of three beacon nodes to calculate distance from $\mathrm{CH}$ node. As given in figure $1, \mathrm{CH}$ and three beacon nodes form triangles and position of each $\mathrm{BN}$ is calculated. Distances $e 1, e 2$ and $e 3$ from $\mathrm{CH}$ to all $\mathrm{BNs}\left(\mathrm{BN}_{\mathrm{A}}, \mathrm{BN}_{\mathrm{B}}\right.$ and $\left.\mathrm{BN}_{\mathrm{C}}\right)$ are calculated as:

$$
\begin{aligned}
& e 1=\text { Signal_Propagation_Speed } *(t 2-t 1) \\
& e 2=\text { Signal_Propagation_Speed } *(t 2-t 1) \\
& e 3=\text { Signal_Propagation_Speed } *(t 2-t 1)
\end{aligned}
$$

Similarly distances $d 1, d 2$ and $d 3$ across BNs are calculated. From geometric properties, the triangle $\mathrm{BN}_{\mathrm{A}} \mathrm{CHBN}_{\mathrm{C}}$ with known position (x, y) and calculated distances $e 1, e 3$ and $e 3$, position $(\mathrm{x} 1, \mathrm{y} 1)$ of $\mathrm{BN}_{\mathrm{A}}$ and position $(\mathrm{x} 3, \mathrm{y} 3)$ of $\mathrm{BN}_{\mathrm{C}}$ are calculated. Similarly, from triangle $\mathrm{BN}_{\mathrm{B}} \mathrm{CHBN}_{\mathrm{C}}$, the position $(\mathrm{x} 2, \mathrm{y} 2)$ of $\mathrm{BN}_{\mathrm{B}}$ is calculated. 


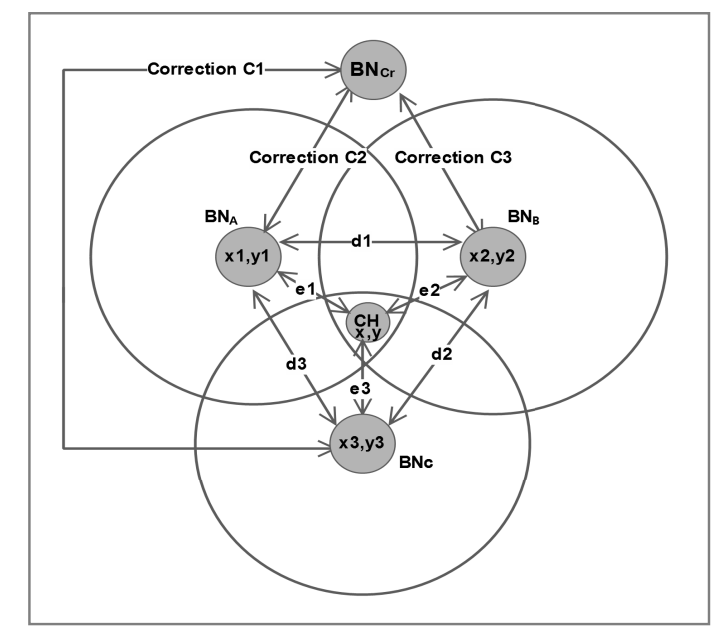

Figure 1. Computation of node position using $\mathrm{CH}, \mathrm{BN}$ and correction $\mathrm{BN}$

Multilateration - Combination of one or more trilateration sets form multilateration. Centroid is computed from each trilateration set and node positions are calculated.

\section{Horizontal Location Position System (H-LPS)}

Sensor cluster is divided into Horizontal Zones (HZs) as given in figure 2. Each row HZ has number of column HZs. After locating first row HZ, the algorithm shifts to second row HZ until all the HZs are covered. In H-LPS, we use four types nodes as (i) CH (ii) BN (iii) Settle node (StN) and (iv) Sensor node (S). We place three beacon nodes horizontally whose location position is not known. These beacon nodes are linked to form a fully connected network. Beacon nodes use $\mathrm{CH}$ as reference node to locate their positions. Nodes exchange information using DV-Hop and DV-Distance techniques to estimate node locations. In APS, if a node is unable to receive packet from beacon node, new packet is re-transmitted and error corrections (if any) are done. In our proposed H-LPS algorithm, we place a new localized beacon node to reconfirm the location of beacon nodes. The new beacon node functions as correction factor for localized beacon nodes. The new node is linked with other localized beacon nodes to form a fully connected network. Figure 3 gives illustration of H-LPS. Let $C H \in H Z 1$. Figure 3(a) indicates how $\mathrm{CH}$ locates BNs. Each BN sends DV-Hop signals to other BNs and calculate node distance and position. Additional localized beacon node, $\mathrm{BNCr}$ is added as correction factor to reconfirm the location of beacon nodes (since their location position form the basic coordinates). Localized beacon node now function as settled node and aid to locate other sensor nodes. This process is repeated in figure 3(c) and 3 (d) until all nodes are localized in HZ2. This process is repeated until HZ3, HZ4, ...., HZn are all localized with in a given cluster. Each cluster has its set of beacon nodes and H-LPS is applied across HZs for all clusters. For some WSN applications all the sensor nodes need not be localized. Let us say for some horizontal zone HZk, localization is not necessary. Then we set the localization flag to FALSE for that particular HZk and remaining nodes are localized. [18] Gives direction based distributed location in WSN. 



Figure 2. WSN Cluster divided into Horizontal Zones

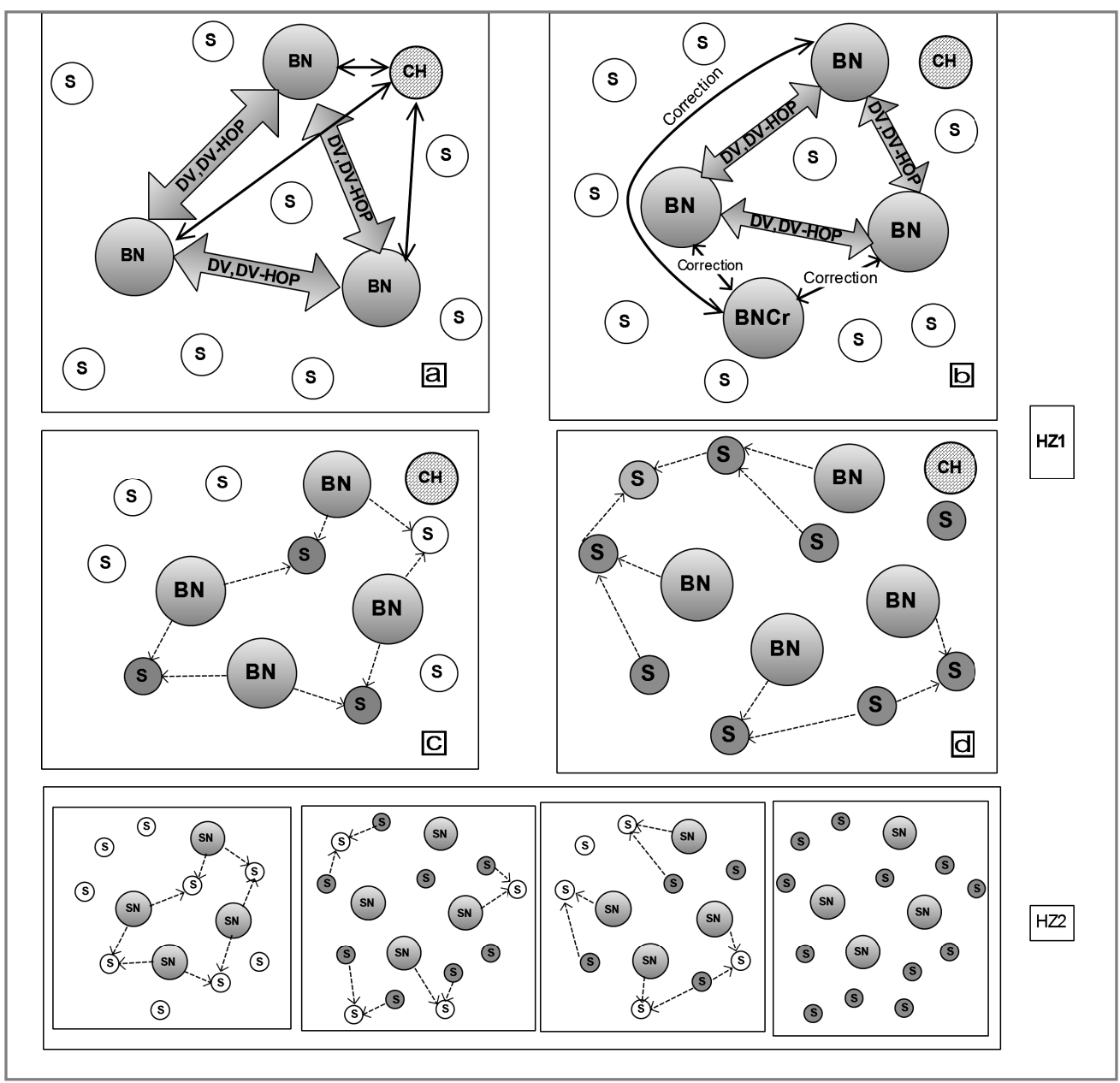

Figure 3. Sequence of steps to locate nodes in HZs with help of BNs and correction B 


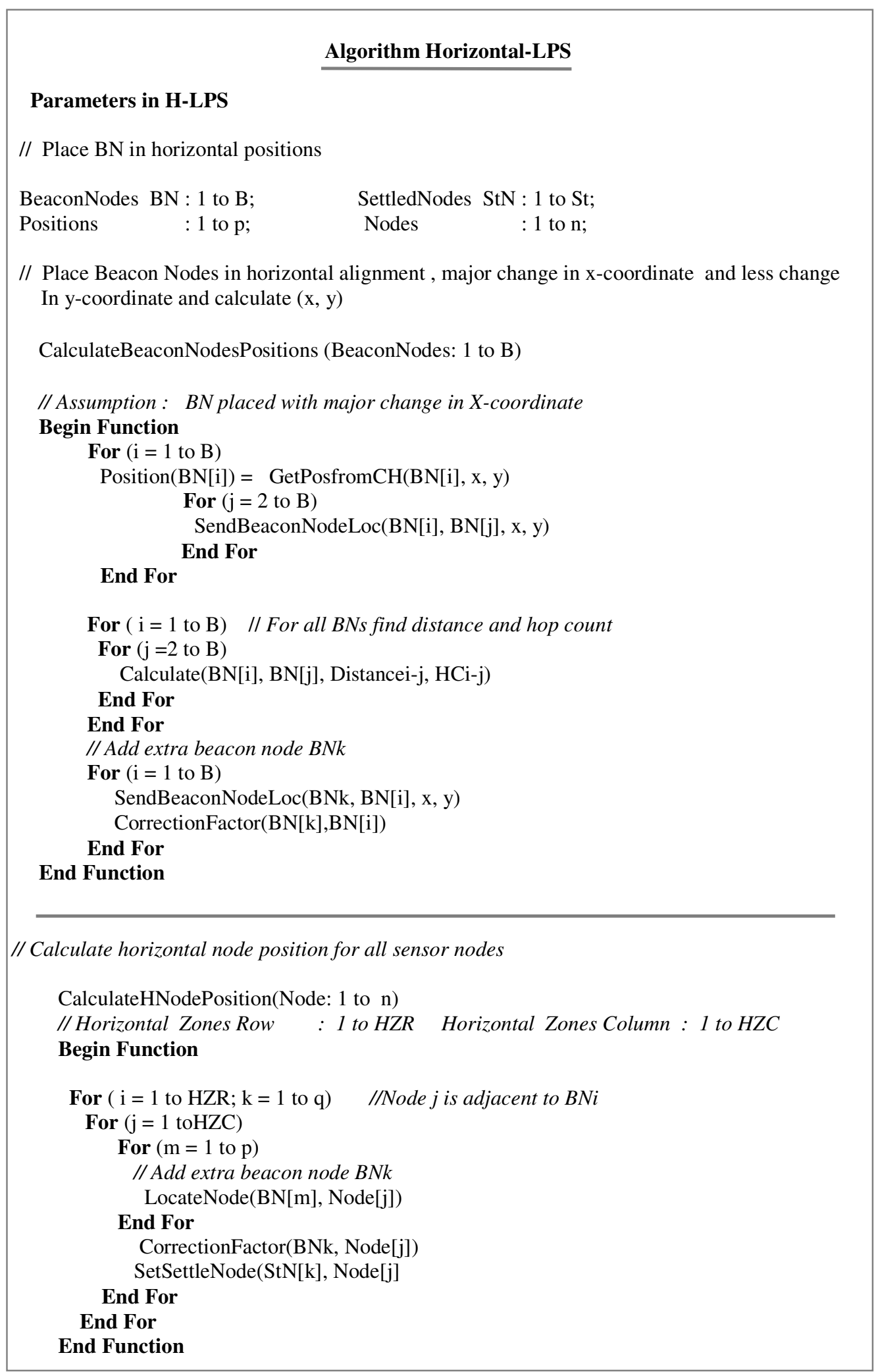




\section{Analysis}

H-LPS location is done horizontally and $\mathrm{CH}$ helps to locate beacon nodes, which further aid to locate sensor nodes. If nodes are to be localized in a particular horizontal zone block, bounded $\mathrm{HZ}$ range [19] is defined between $(\mathrm{x} 1, \mathrm{y} 1)$ and $(\mathrm{x} 2, \mathrm{y} 2)$. Nodes that do not belong to bounded $\mathrm{HZ}$ range are not localized and thus save WSN localization cost.

To split cluster $\mathrm{C}$ into $n \mathrm{HZs}$ during time interval $t 1$ to $t 2$ is given as:

$$
H Z_{n}=\int_{t 1}^{t 1} \operatorname{SplitCluster}(C, n H Z)
$$

Time to locate nodes in a $\mathrm{HZ}$ within a cluster $\mathrm{C}$ is given as:

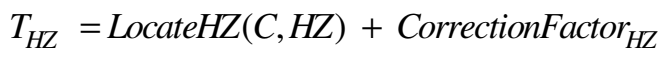

Time to locate nodes in all $\mathrm{HZs}$ within a cluster $\mathrm{C}$ is given as:

$$
T_{H Z[1-n]}=\sum_{H Z=1}^{n}\left(\text { LocateHZ}_{\left.(C, H Z)+\text { CorrectionFactor }_{H Z}\right)}\right.
$$

\section{Vertical Location Position System (V-LPS)}

In our proposed Vertical-LPS, unknown sensor nodes are located vertically. Sensor cluster is divided into Vertical Zones (VZs). Each column VZ has number of row VZs as given in figure 4. After locating first column VZ, the algorithm shifts to second column VZ until all the VZs are covered. [20] Gives self-configuring localization systems. In V-LPS, similar to H-LPS we place three beacon nodes vertically. These beacon nodes are linked to form a fully connected network. Nodes exchange information using DV-Hop and DV-Distance techniques to estimate their location. Similar to H-LPS algorithm, we place a new localized beacon node to reconfirm the location of beacon nodes. New beacon node function as correction factor for localized beacon nodes. The new node is linked with other localized beacon nodes to form a fully connected network. This node exchange information with all the other localized beacon nodes using DV-Hop and DV-Distance and confirms the location of nodes. In V-LPS, the localization algorithms extend in vertical direction across various VZs until all the nodes are localized as given in figure 5. Remaining functionality is similar to H-LPS. Algorithm of V-LPS is similar to H-LPS. [21] Gives distributed weighted multidimensional scaling for node localization in sensor networks.

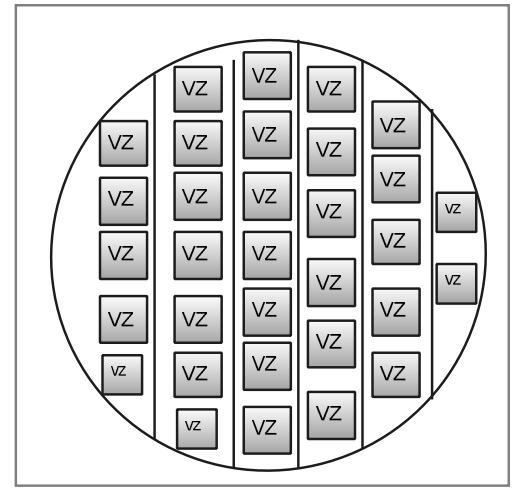

Figure 4. WSN Cluster divided into Vertical Zones 
International Journal of Wireless \& Mobile Networks (IJWMN) Vol.2, No.4, November 2010



Figure 5. Sequence of steps to locate nodes in VZs with help of BN and correction BN.

\section{Analysis}

V-LPS location is done vertically and $\mathrm{CH}$ helps to locate beacon nodes, which further aid to locate sensor nodes. If nodes are to be located in a particular vertical zone, bounded VZ range is defined between $(\mathrm{x} 1, \mathrm{y} 1)$ and $(\mathrm{x} 2, \mathrm{y} 2)$. Nodes that do not belong to bounded VZ range are not localized and thus save WSN localization cost.

To split cluster C into $n$ VZs during time interval $t 1$ to $t 2$ is given as:

$$
V Z_{n}=\int_{t 1}^{t 1} \operatorname{SplitCluster}(C, n V Z)
$$

Time to locate nodes in a $\mathrm{VZ}$ within a cluster $\mathrm{C}$ is given as:

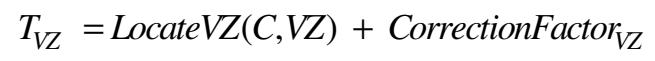

Time to locate nodes in all VZs within a cluster $\mathrm{C}$ is given as: 




Localization of wireless sensor networks with a mobile beacon is given in [22].

\section{Simulations}

Simulations are done using NS-2 and parameters are given in table 1. The simulation comparisons are done for APE, RPE and DPE distributed localization techniques. Simulation parameters considered for H-LPS and V-LPS algorithms are number of sensor nodes, percentage zone localization error, simulation time and addition of beacon nodes.

Table 1. Simulation Parameters

\begin{tabular}{|c|c|c|c|}
\hline Parameter & Value & Parameter & Value \\
\hline Simulation Area & $1500 \mathrm{~m} \times 1500 \mathrm{~m}$ & Traffic Type & CBR \\
\hline Simulation Time & $700 \mathrm{~s}$ & $\begin{array}{c}\text { Number of } \\
\text { Sources }\end{array}$ & 9 \\
\hline Number of nodes & 250 & Packet size & $512 \mathrm{Bytes}$ \\
\hline $\begin{array}{c}\text { Number of Beacon } \\
\text { Nodes }\end{array}$ & 50 (Maximum) & Arrival rate & $20 \mathrm{pkt} / \mathrm{sec}$ \\
\hline Node Placement & Random & Node range & $250 \mathrm{~m}$ \\
\hline
\end{tabular}

We simulated the proposed algorithm H-LPS and V-LPS for localization in WSN with existing localization techniques. Figure 6 gives comparison of percentage zone location position error with varying number of sensor node for the proposed H-LPS, V-LPS and existing localization techniques APS, RPE and DPS. As the number of sensor nodes increase, zone localization error increases and beyond a threshold localization error becomes constant. For existing distributed localization techniques, average localization error gets stabilized between $0.45 \%$ and $0.55 \%$. For H-LPS and V-LPS average localization error gets stabilized between $0.32 \%$ and $0.35 \%$. Percentage zone position error can further be minimized if the numbers of nodes are localized in the direction of packet traversal. During simulations in bounded zone localization for H-LPS and V-LPS, further reduction in percentage zone location position error was notified.

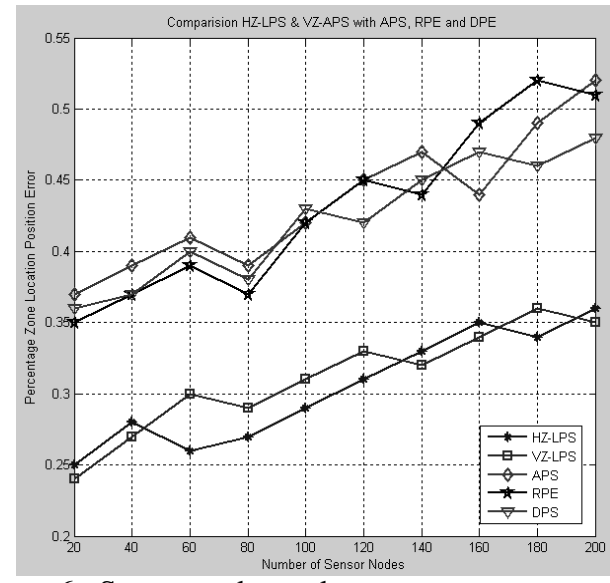

Figure 6. Sensor nodes and percentage zone location position error

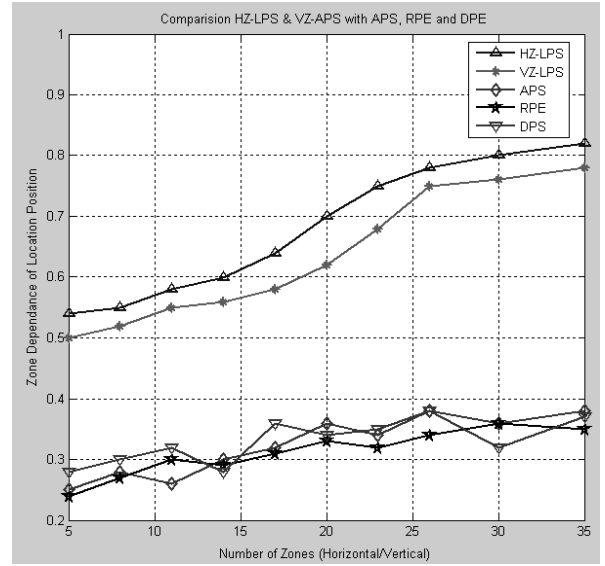

Figure7. Number of zones (HZ/VZ) and zone dependence location position

This clearly indicates an improvement in H-LPS and V-LPS over existing localization techniques. Figure 7 gives simulation results for localization zone dependence. Localization zone 
dependence is less in existing protocols compared to our proposed algorithms. Due to zone dependence, nodes within a given area are localized and remaining nodes are not localized. Simulation results indicate localization zone dependence factor is 0.30 to 0.25 in existing localization techniques and 0.80 to 0.85 in our proposed H-LPS and V-LPS techniques. This saves WSN localization cost and improves the performance of localization techniques. Figure 8 gives results for simulation time and average percentage of zones localized (with nodes localized in each zone). As the simulation time increases, more number of zones are localized and nodes in each zone are identified by communication and network parameters. Percentage zone localization is enhanced in H-LPS and V-LPS compared to APE, RPE and DPS. Figure 9 gives simulation results for increase in beacon nodes. As the number of beacon nodes increase, percentage localization error decreases for all localization algorithms (existing and proposed). Clearly, simulation results indicate zone localization error is less in H-LPS and V-LPS compared to existing localization techniques.



Figure 8. Simulation time and average percent zone location.

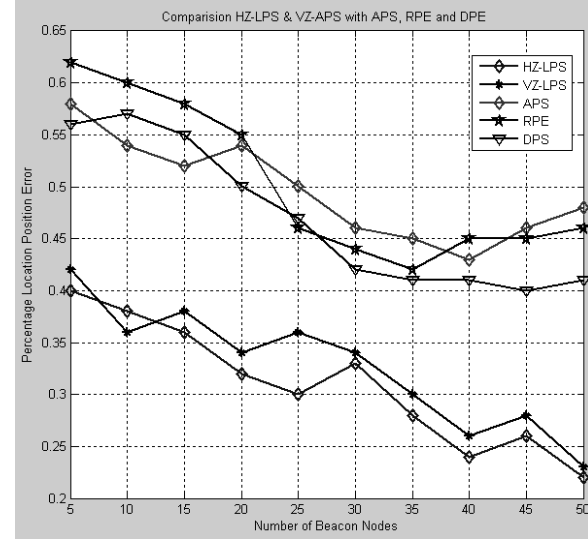

Figure 9. Number of Beacon nodes and percentage location position error

\section{CONCLUSIONS}

In this article we propose distributed localization algorithms, H-LPS and V-LPS with position known Cluster Head $(\mathrm{CH})$ and position unknown beacon nodes. Nodes are assumed to be static. Using traditional trilateration technique, node position is estimated. We add extra position known beacon node to reconfirm the localized beacon nodes. This eliminates percentage localization error to $70 \%$. The main advantage of zone based localization is, nodes belonging to bounded zone are localized and nodes that do not belong to bounded zone are not localized since they do not participate in WSN computing and thus save localization cost. Simulations results indicate better performance for zone based localization in H-LPS and V-LPS compared to existing localization techniques like Ad hoc Positioning System (APS), Recursive Positioning Estimation (RPE) and Directed Positioning Estimation (DPE). Extension of this work can be implementation of dynamic node structure for H-LPS and V-LPS.

\section{REFERENCES}

[1] Guoqiang Mao and Baris Fidan (2009), "Localization Algorithms and Strategies for Wireless Sensor Networks", Information Science Reference (Imprint of IGI Global), UK.

[2] Masoomeh Rudafshani and Suprakash Datta (2007), "Localization in Wireless Sensor Networks", IPSN'07, April 2007, pp. 51-60.

[3] Guoqiang Mao, Barıs Fidan and Brian D.O. Anderson (2007), "Wireless sensor network localization techniques", Elsevier Computer Networks, 2007 Volume 51, pp. 2529-2553. 
[4] Azzedine Bouerche, Horacio A.B.F.Oliveira, Eduardo F.Nakamura and Antonio A.F.Loureiro (2007), "Localization Systems for Wireless Sensor Networks", IEEE Wireless Communication Magazine, December 2007, pp. 6-12.

[5] L. Doherty, K. S. J. Pister, and L. El Ghaoui (2001), "Convex position estimation in wireless sensor networks", Proceedings of IEEE INFOCOM, volume 3, April 2001, pp. 1655 - 1663.

[6] Y. Shang, W. Ruml, Y Zhang, and M. Fromherz (2004), "Localization from connectivity in sensor networks", IEEE Transactions on Parallel and Distributed Systems, no. 11, volume 15, pp. $961-974$.

[7] C. Savarese, J. M. Rabaey, and J. Beutel (2001), "Location in distributed ad - hoc wireless sensor networks", Proceedings of 2001 International Conference on Acoustics, Speech, and Signal Processing (ICASSP '01), May 2001, pp. 2037 - 2040.

[8] Gwo-Jong Yu and Shao-Chun Wang (2008), "A Hierarchical MDS-based Localization Algorithm for Wireless Sensor Networks", Journal of Information Technology and Applications, 2008, Volume 2, No. 4, pp. 197-202.

[9] Cesare Alippi, Giovanni Vanini (2006), "A RSSI-based and calibrated centralized localization technique for Wireless Sensor Networks", Proceedings of Fourth IEEE International Conference on Pervasive Computing and Communications Workshops (PERCOMW'06), March 2006, pp. 301-305.

[10] J. Albowicz, A. Chen, and L. Zhang (2001), "Recursive position estimation in sensor networks", The 9th International Conference on Network Protocols, November 2001, pp. 35-41.

[11] D. Niculescu and B. Nath (2001), "Ad - hoc positioning system", Proceedings of 2001 IEEE Global Communications Conference (Globecom ' 2001), November 2001, pp. 2926 - 2931.

[12] C. Savarese, K. Langendoen, and J. Rabaey (2002), "Robust positioning algorithms for distributed ad-hoc wireless sensor networks", Proceedings of USENIX Technical Annual Conference, 2002, pp. $317-328$.

[13] Peng Aiping, Guo Xiaosong, Cai Wei, Li Haibin, (2009) "A Distributed Localization Scheme for Wireless Sensor Networks Based on Bounding Box Algorithm", IEEE The Ninth International Conference on Electronic Measurement \& Instruments (ICEMI'2009), pp. 984-988.

[14] T. He, C. Huang, B. Blum, J. Stankovic, and T. Abdelzaher (2003), "Range-Free Localization Schemes in Large Scale Sensor Networks", Proceedings of the Ninth Annual international conference on Mobile computing and networking (MobiCom 2003), pp. 81 - 95.

[15] H. A. B. F. Oliveira, E. F. Nakamura, A. A. F. Loureiro, and A. Boukerche (2005), "Directed position estimation: A recursive localization approach for wireless sensor networks", Proceedings of the 14th IEEE International Conference on Computer Communications and Networks (IC3N '05), October 2005, pp. 557-562.

[16] Azzedine Boukerche (2009), "Algorithms and Protocols for Wireless Sensor Networks", John Wiley \& Sons, Inc., New Jersey, USA.

[17] Amitangshu Pal (2010), "Localization Algorithms in Wireless Sensor Networks: Current Approaches and Future Challenges", Network Protocols and Algorithms, Volume 2, No. 1.

[18] Sheng-Shih Wang, Kuei-Ping Shih and Chih-Yung Chang (2007), "Distributed direction-based localization in wireless sensor networks", Elsevier Computer Communications, Volume 30, 2007, pp. 1424-1439.

[19] Lei Zhang and Binwei Deng (2009), "A New Range-based Localization Algorithm for Wireless Sensor Networks", International Colloquium on Computing, Communication, Control, and Management (CCCM 2009), pp. 111-114.

[20] Nirupama Bulusu,John Heidemann, Deborah Estrub and Tommy Tran (2004),"Self-Configuring Localization Systems:Design and Experimental Evaluation", ACM Transactions on Embedded Computing Systems, Vol. 3, No. 1, February 2004, pp. 24-60.

[21] J. A. Costa , N. Patwari , and A. O. Hero (2006) , “ Distributed weighted - multidimensional scaling for node localization in sensor networks", ACM Transactions on Sensor Networks (TOSN), volume 2 no. 1, February 2006, pp. 39 - 64.

[22] M. L. Sichitiu and V. Ramadurai (2004), "Localization of wireless sensor networks with a mobile beacon", Proceedings of the 1st IEEE International Conference on Mobile Ad Hoc and Sensor Systems (MASS 2004), October 2004, pp. 174-183. 


\section{AUTHOR BIOGRAPHIES}

M. Bala Krishna is assistant professor in University School of Information Technology, Guru Gobind Singh Indraprastha University, Delhi, INDIA. His areas of interest include architecture and protocols in wireless networks and mobile computing. He had worked in various research projects of embedded systems design and wireless communication networks. He is working in the area of wireless sensor and ad hoc networks, and embedded system applications. He is an active member of various reputed technical organizations.

M. N. Doja is professor in Department of Computer Engineering, Jamia Millia Islamia, Delhi, INDIA. His research areas include computer networks and mobile wireless networks, AI and 4G communications. $\mathrm{He}$ has various publications in soft computing techniques. He is author of various books and has publications in various national and international conferences and journals. He received awards from many prestigious institutions. He is chair and co-chair of various technical organization committees at national and international levels. 